\title{
Enzymes and Substrates Are Balanced at Minimal Combined Mass \\ Concentration in vivo
}

Hugo Dourado ${ }^{1,2}$, Veronica G. Maurino ${ }^{2,3}$ \& Martin J. Lercher ${ }^{1,2 *}$

${ }^{1}$ Institute for Computer Science and Department of Biology, Heinrich Heine University, D-40225 Düsseldorf, Germany.

${ }^{2}$ Cluster of Excellence on Plant Sciences (CEPLAS), D-40225 Düsseldorf, Germany.

${ }^{3}$ Institute of Developmental and Molecular Biology of Plants, Plant Molecular Physiology and Biotechnology Group, Heinrich Heine University, D-40225 Düsseldorf, Germany.

*Corresponding author: Martin Lercher, martin.lercher@hhu.de

\begin{abstract}
A fundamental problem in biology is how cells organize their resource investment. Cellular metabolism, for example, typically involves hundreds of enzymes and metabolites, but it is unclear according to which principles their concentrations are set. Reasoning that natural selection will drive cells towards achieving a given physiological state at minimal cost, we derive a general equation that predicts the concentration of a metabolite from the concentration of the most abundant and costly enzyme consuming it. Simulations of cellular growth as well as experimental data demonstrate that costs are approximately proportional to molecular masses. For effectively irreversible reactions, the cell maximizes its metabolic efficiency by investing equally into substrate and unbound enzyme molecules. Without fitting any free parameters, the resulting model predicts in vivo substrate concentrations from enzyme concentrations and substrate affinities with high accuracy across data from $E$. coli and diverse eukaryotes $\left(R^{2}=0.79\right.$, geometric mean fold-error 1.74$)$. The corresponding organizing principle - the minimization of the summed mass concentrations of solutes - may facilitate reducing the complexity of kinetic models and will contribute to the design of more efficient synthetic cellular systems.
\end{abstract}




\section{Introduction}

Optimality principles have been used to predict diverse complex cellular properties (1), such as the efficient use of metabolic networks to convert nutrients to biomass (2), the regulation of ribosome content during bacterial growth (3), or the partitioning of membrane occupancy between transporters and the electron transport chain (4). It is likely that in most cells, natural selection has favoured metabolic efficiency, i.e., a near optimal cost/benefit relationship for each active biochemical reaction. While the benefit corresponds to the maintenance of a desired reaction rate, the cost lies in the concentrations of the molecules supporting the reaction (5-7).

It is still unclear what factors dominate the costs associated with concentrations of individual proteins or other molecule types. It has recently been argued that the major cost factor of metabolic reactions stems from protein production costs (8-11), including the allocation of cellular resources such as ribosomes and the consumption of ATP and carbon. Other recent publications have stressed the importance of volumetric costs related to the limited solvent capacity of cellular compartments. The summed volume concentration of solutes cannot exceed critical values, beyond which adequate diffusion would break down $(5,6)$. Based on the limited solvent capacity, it has been proposed that intermediate metabolite concentrations are minimized by natural selection $(5,6,12)$. However, the majority of the cellular volume is occupied by proteins, while metabolites account for only a minor fraction; in E. coli, proteins outweigh metabolites 16:1. (13) Accordingly, some authors have argued that the solvent capacity limits enzyme rather than metabolite concentrations (14-16), a phenomenon termed macro-molecular crowding. Limiting the total enzyme concentration while maximizing biomass production indeed allows to predict different variants of overflow metabolism, such as the Crabtree and Warburg effects, at least qualitatively (14-16).

While most previous researchers $(8-11,14-16)$ considered only the cost of enzymes, reaction rates are jointly determined by enzymes and their substrates. Thus, we propose that the action of natural selection on intracellular concentrations can only be appreciated fully when we consider costs incurred through both types of molecules simultaneously; this argument holds both for production costs (reflecting nutrient consumption) and volumetric costs (reflecting consumption of the limited solvent capacity). 


\section{Results}

To examine the consequences of this hypothesis on the balance between substrate and enzyme concentrations for individual reactions, we first consider an enzyme that converts a single substrate irreversibly into a product following Michaelis-Menten kinetics, where the substrate is not consumed by any other reaction. The reaction rate $v$ is then proportional to the concentrations of unbound enzyme $\left(E_{\text {free }}\right)$ and substrate $\left(M_{\text {free }}\right)$, parameterized by the enzyme's turnover number $k_{c a t}$ and Michaelis constant $K_{m}$ (inversely related to the enzyme's substrate affinity): $v=\frac{k_{c a t}}{K_{m}} E_{\text {free }} M_{\text {free }}$. The concentration of unbound enzyme molecules is approximated as a function of the total concentrations of enzyme $(E)$ and substrate $(M)$ as $E_{\text {free }} \approx \frac{E}{\left(1+M / K_{m}\right)}$; with $M_{\text {free }} \approx M$, rearrangement results in the standard Michaelis-Menten equation.

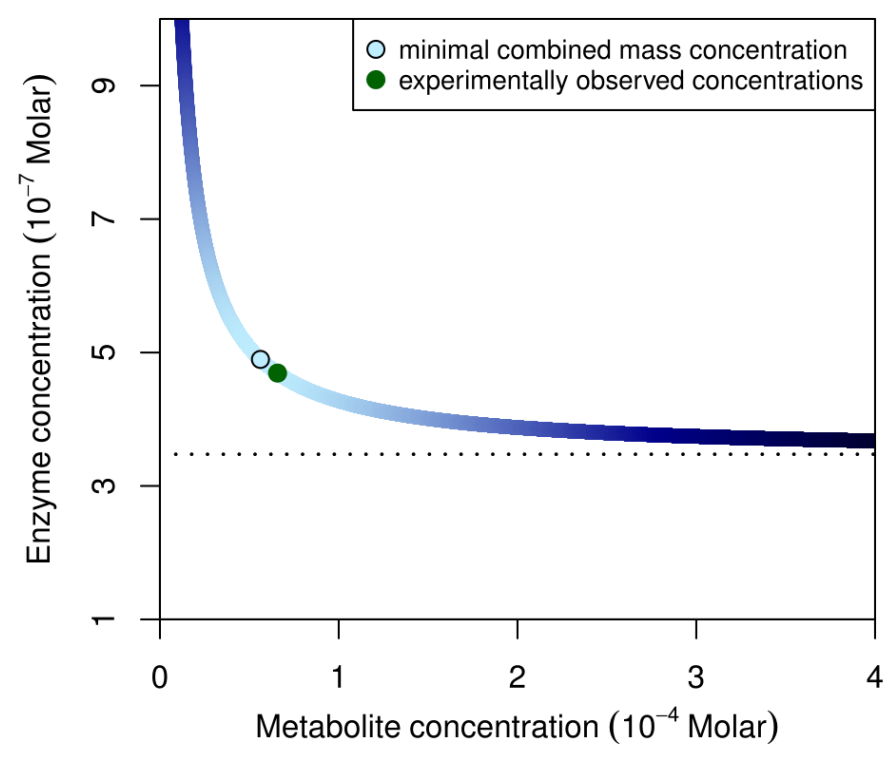

Figure 1. For each Substrate concentration $M$, there is one enzyme concentration $E$ that supports a given reaction rate $v$. The curve shown is for the GMP reductase enzyme (GuaC) and its substrate GMP in E. coli, color-coded according to the summed mass concentration of GuaC and GMP. The combination resulting in the smallest summed mass concentration is indicated by the circle; the green dot indicates the in vivo combination observed for $E$. coli growing on glycerol $(23,24)$.

A reaction rate $v$ per unit volume "demanded" by the current cellular state can be achieved in multiple ways: for each non-zero substrate concentration $M$, there is exactly one enzyme concentration $E$ (and a corresponding $E_{\text {free }}$ ) that results in the desired rate (Figure 1). The costs 
associated with the enzymatic reaction will depend approximately linearly on $E$ and $M$ at least for small concentration changes (11); they can thus be expressed through the specific costs of enzyme and substrate molecules, $c_{E}$ and $c_{M}$, respectively. Under natural selection for metabolic efficiency, the cell is expected to choose the combination of concentrations that minimizes the summed costs of enzyme and substrate. Because the reaction rate depends equally on the concentrations of unbound enzyme and metabolite, the optimally efficient metabolic state invests equally into these two types of molecules:

$$
c_{E} E_{\text {free }}=c_{M} M
$$

(for a formal derivation and generalizations, see SI Text). Converting to total enzyme concentration $E$ and defining the cost ratio of enzyme and substrate molecules $a:=c_{E} / c_{M}$, we can rewrite this as

$$
a E=M\left(1+\frac{M}{K_{m}}\right)
$$

Strikingly, this optimal relationship between enzyme and substrate concentration depends only on the Michaelis constant $K_{m}$ and on the cost ratio $a$, but is independent of reaction rate and turnover number.

Reality is of course more complex than the irreversible single-substrate reaction discussed so far. Cellular metabolism forms a highly interconnected network: many reactions involve more than one substrate, and many metabolites are consumed by more than one enzyme. When considering metabolic costs, we must therefore account for all metabolite and enzyme concentrations simultaneously. Using convenience kinetics to approximate general reaction kinetics (17), it is easy to show that we can consider each metabolite separately as long as the relevant reactions are effectively irreversible (SI Text). The resulting equation for a single metabolite relates its concentration to the concentrations of all enzymes consuming it, mediated by the corresponding Michaelis constants and the relative costs. If one of the enzymes dominates this mathematical relationship, the terms corresponding to the other enzymes can be neglected; typically, the "dominant" enzyme has the largest $a E$, i.e., the highest total cost among all enzymes consuming the metabolite. This approximation results in an equation identical to Eq. (2) that considers only the dominant enzyme (SI Text). Note that the dominant enzyme in our terminology may not be 
the enzyme responsible for the highest turnover of the metabolite, but is the one with the highest total cost in the current metabolic state.

Generalizations to multifunctional enzymes, stoichiometries other than $1: 1$, reversible reactions, and Hill kinetics are derived and summarized in the SI Text. All considered cases are well approximated by Eq. (2) or its generalization to other stoichiometries.

To compare Eq. (2) to experimental data, we first need to determine the cost ratio $a$ of enzymes and metabolites. Indirectly, such costs can be observed as growth rate reductions in experiments that force bacteria to overexpress unneeded proteins or metabolites $(7,10,18,19)$. However, these observations cannot be directly transformed into cost estimates: forced overexpression may cause major cellular reorganizations (20), and the reported effects are unlikely to represent metabolically efficient states (21).

To nevertheless explore different approximations to the enzyme-metabolite cost ratio, we utilize a minimalistic in silico cell model (15). Our model is comprised of a small set of transport and enzymatic reactions that follow Michaelis-Menten kinetics and convert two nutrients (termed C and $\mathrm{N}$ ) to precursors for the production of proteins, including a ribosome, and of lipids (Fig. S1a; for detailed methods see SI Text). The simulations search for a combination of protein and metabolite concentrations that maximizes balanced growth, where all cellular components are reproduced in proportion to their concentrations. This model fully accounts for molecular production costs as well as a solvent capacity limit on the summed volume concentration of all intracellular solutes. Model parameters are the external nutrient concentrations, kinetic constants of the biochemical reactions, protein and metabolite masses, and the cytosolic solvent capacity. To simulate a limiting nutrient, we considered 1000 random parameterizations with low external concentrations of $\mathrm{N}$ while allowing unlimited uptake of $\mathrm{C}$.

We first tried to approximate cost ratios through the relative molecular yield of enzyme and metabolite production from the limiting nutrient, $a_{N}$, reflecting direct production costs. This resulted in a Pearson correlation of $R^{2}=0.78$ between the simulated metabolite concentrations and those predicted from enzyme concentrations via Eq. (2) (Fig. 2B; geometric mean fold-error $G M F E=1.83$, considering only molecules that require $\mathrm{N}$ for their production). For comparison, we repeated this analysis using molecular weights as a proxy for the relative costs of enzymes and metabolites, $a_{m}$. Molecular weight appears a reasonable first approximation for production costs of different molecule species, even if this measure does not account for differences in 
atomic composition, cofactor utilization, and length of required production pathways. Moreover, molecular volumes correlate strongly with molecular weights (22), and thus $a_{m}$ directly reflects relative volumetric costs. Employing the mass ratio $a_{m}$ in Eq. (2) resulted in significantly improved predictions (Fig. $2 \mathrm{~A} ; R^{2}=0.85, G M F E=1.68$; empirical $P$-value for the superiority of predictions from $a_{m}$ compared to $a_{N}: P<10^{-15}$ ). Strikingly, the superiority of molecular mass as a proxy for total cost becomes most pronounced when we consider only those metabolites made up exclusively of the limiting nutrient $\mathrm{N}\left(a_{m}: G M F E=2.52 ; a_{N}: G M F E=4.06\right)$. Simulations where C and $\mathrm{N}$ are equally growth limiting lead to very similar results (Fig. S1).

a

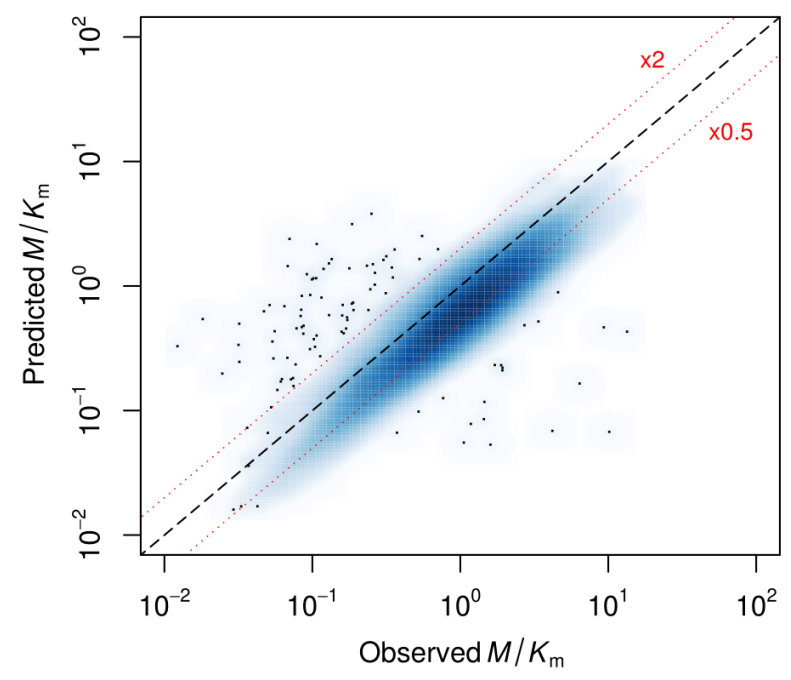

b

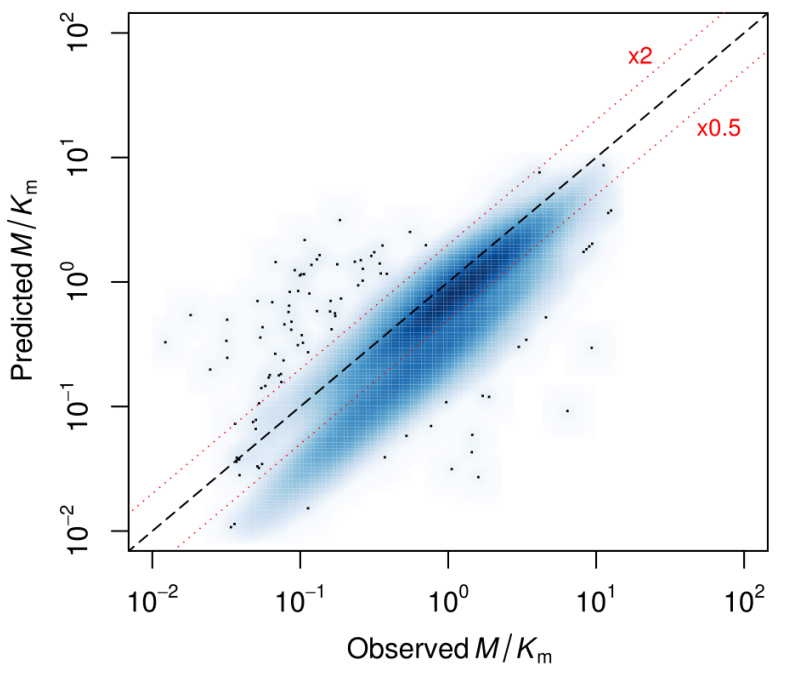

Figure 2. Eq. (2) predicts metabolite concentrations from enzyme concentrations observed in the minimal in silico cell accurately when relative enzyme/metabolite costs are approximated through the mass ratio $a_{m}\left(R^{2}=0.85, G M F E=1.68\right)(\mathrm{a})$, but less accurately when approximated through molecular yields for production from the limiting nutrient $\left(R^{2}=0.78, G M F E=1.83\right)$ (b). Each smoothed scatter plot summarizes results across all intracellular reactions for 1000 random model parameterizations; colour intensity is proportional to data density, points shown individually are outliers. The black dashed lines indicate the expected identity; the upper and lower red dotted lines indicate deviations of $\times 2$ and $\times 0.5$, respectively.

We conclude that the behaviour of intracellular concentrations in the in silico cell is well described by Eq. (2) when approximating relative costs through mass ratios. Can the same model also predict the relationship between enzyme and metabolite concentrations in vivo? Experimental data for absolute intracellular concentrations are only available for a limited number of enzyme-metabolite pairs in Escherichia coli $(23,24)$ and in the yeast Saccharomyces 
cerevisiae $(25,26)$, and for isolated reactions in red blood cells (27) and in the green alga Chlamydomonas reinhardtii (28). Application of Eq. (2) requires knowledge of $K_{m}$ for the dominant enzyme, further reducing the sample size.

All available data accurately conforms to Eq. (2) (Fig. 3a,c). Dominant enzyme concentrations together with $K_{m}$ values and molecular mass ratios are sufficient to predict in vivo metabolite concentrations with a Pearson correlation of $R^{2}=0.79\left(P<10^{-16}\right)$ and a geometric mean fold-error $G M F E=1.74$ across the combined E. coli and eukaryotic data (Fig. 3b,d). We conclude (i) that the costs of intracellular concentrations can be approximated through molecular masses not only in the in silico cell but also in vivo, and (ii) that biological cells optimized for metabolic efficiency balance their enzyme and metabolite concentrations accordingly. The metabolite concentrations (26) in yeast were measured for a slightly different strain and on a different growth medium compared to the protein concentration measurements (25); this discrepancy may contribute to the larger deviation between predictions and measurements in the yeast data compared to other cell types (Fig. 3). Both the E. coli and the S. cerevisiae data includes tRNAcharging reactions (Fig. S2), emphasizing the applicability of the proposed relationships also to non-metabolic enzymatic reactions.

E. coli protein expression patterns may not be geared towards maximal metabolic efficiency in many conditions (29). When considering only the E. coli data for the two carbon sources most likely to be optimized for metabolic efficiency - those with the highest growth rates, glucose and fructose (23) - we obtain $R^{2}=0.90\left(P=10^{-8}\right)$ and $G M F E=1.51$, compared to $R^{2}=0.79\left(P=10^{-16}\right)$ and $G M F E=1.67$ when considering $E$. coli data across all growth conditions. Indeed, for most reactions, enzyme concentrations show little variation across conditions, while metabolite concentrations are much more variable (Fig. S2). This might indicate that for each of these reactions, enzyme levels are optimally regulated for growth on glucose and/or fructose regardless of the condition. This pattern would be consistent with an evolutionary strategy that shortens the lag-phase at the transition from an unpreferred to a preferred carbon source, minimizing associated changes in protein expression at the cost of non-optimal expression in unpreferred conditions (30). 

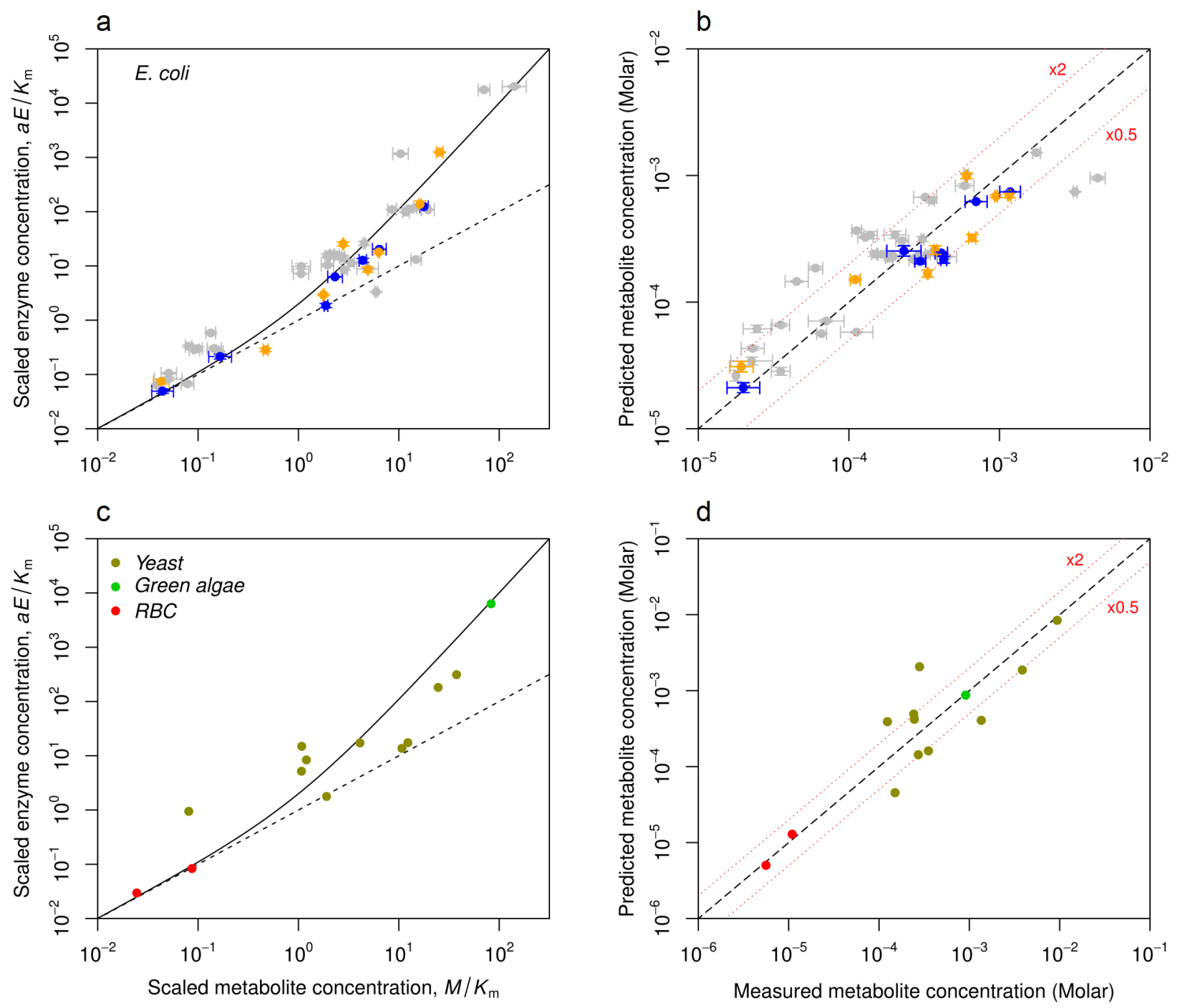

Figure 3. (a) Eq. (2) holds accurately across available data from E. coli (orange: growth on glucose; blue: growth on fructose; grey: growth on other carbon sources). Concentrations of metabolites (24) and enzymes (23) are scaled as $x=M / K_{m}$ and $y=a_{m} E / K_{m}$ with molecular mass ratio $a_{m}$, such that all predictions follow $y=x(1+x)$ (solid line). The diagonal (dotted line) indicates equal cellular mass concentrations of metabolite and enzyme $\left(a_{m} E=M\right)$. Error bars are reported measurement errors. (b) Predicted vs. observed E. coli metabolite concentrations (Pearson's correlation between log-transformed values $R^{2}=0.79, P=10^{-16}$; $G M F E=1.67$; data restricted to growth on glucose and fructose: $R^{2}=0.90, P=6 \times 10^{-8}$, $G M F E=1.51)$. The dashed line indicates the expected identity. The upper and lower red dotted lines indicate deviations of $\times 2$ and $\times 0.5$, respectively. For the $x$-axis, error bars are reported measurement errors; for the $y$-axis, error bars reflect the expected standard deviation based on reported measurement errors for enzyme concentrations. See Fig. S2 for the E. coli raw data and individual growth conditions. (c) Eq. (2) holds accurately across available data from diverse eukaryotes (yellow: yeast (Saccharomyces cerevisiae); red: red blood cell; green: green alga (Chlamydomonas reinhardtii)). Concentrations are scaled as in panel (a). (d) Predicted vs. observed eukaryotic metabolite concentrations $\left(R^{2}=0.80\right.$, $\left.P=4 \times 10^{-5} ; G M F E=2.06\right)$. 
Red blood cells have little room for enzymatic reactions, as hemoglobin makes up 97\% of their cellular dry weight (31). Consequently, metabolite concentrations are much below $K_{m}$ in this cell type (27), and reaction rates are reduced to $2-8 \%$ of their values at saturation. As predicted by the asymptotic behaviour of Eq. (2), the total cellular mass of an enzyme and its substrate becomes very similar in this case $\left(a_{m} E \approx M\right.$; Fig. $\left.3 \mathrm{c}\right)$ : when most enzymes are in their unbound state, optimal investment is distributed equally across enzyme and substrate molecules. At the other end of the spectrum, Rubisco molecules in the green alga $C$. reinhardtii are highly saturated $\left(M=83 \times K_{m}\right)$ and outweigh their substrate ribulose 1,5 bisphosphate (RuBP) 75:1, (28) a relationship still accurately predicted by Eq. (2). While all other enzyme-substrate pairs considered here correspond to cytosolic reactions, Rubisco is located in algal chloroplasts, emphasizing the applicability of our results to cellular compartments other than the cytosol.

Several of the reactions included in Fig. 3 are reversible (Table S1), and it is not obvious that Eq. (2) can be applied in these cases. For one of the reactions, Fumarase A (fumA) consuming fumarate (FUM) in E. coli, we also have concentration measurements for the product (L-malate) as well as the full kinetic constants of the reversible Michaelis-Menten equation (32). In all assayed conditions, the ratio of product/substrate concentrations is much smaller than the equilibrium constant for this reaction, $K_{e q}=11.0$ (Table S1). As long as the product is not strongly saturating the enzyme, which is the case for the majority of conditions $\left(P / K_{m}{ }^{P} \leq 2.5\right)$, the predictions derived from Eq. (2) do not differ substantially from those derived with its equivalent for reversible reaction kinetics, Eq. (S50). During growth on succinate, however, the product is strongly saturating $\left(P / K_{m}{ }^{P}=15.0\right)$; here, employing the reversible prediction reduces the mean fold-error from 4.71 to 1.22 (Fig. S3). The overall excellent match between observed metabolite concentrations and those predicted via Eq. (2) (Fig. 3) suggests that the FUM-fumA case is representative for reversible reactions in general: across most growth conditions, generally reversible reactions may be rendered effectively irreversible by metabolite concentrations that provide an adequate thermodynamic driving force. This notion agrees with previous considerations of the effect of thermodynamic driving forces on expected metabolite concentrations $(11,12)$, and with a detailed analysis of the relationship between metabolite concentrations and measurements of forward to backward flux ratios (26).

Our hypothesis of natural selection on minimal cellular reaction costs makes two specific predictions when considering one metabolite across different growth conditions. First, if the dominant enzyme remains the same across conditions, then we expect the corresponding points 
to follow the prediction line from Eq. (2), with different positions corresponding to differences in the flux through the reaction across conditions. This can be seen for galactose-1-phosphate uridylyltransferase (GalT): this enzyme is expressed at very low levels except in growth on galactose, where its substrate shows a correlated increase (Fig. S2e). Second, if an enzyme is dominant for a given substrate only in some of the assayed conditions, we expect to see strong deviations from Eq. (2) in those conditions where other enzymes that consume the same substrate become more costly (have higher $a_{m} E$ ). This can also be observed in the $E$. coli dataset. 1-phosphofructokinase (FruK) is dominant for beta-D-fructofuranose 1-phosphate (F1P) during growth on fructose and predicts the observed F1P concentration within a factor of 1.71. However, FruK contributes only $2.0 \%$ and $2.6 \%$ to the total enzyme mass consuming this substrate on acetate and glucose, respectively. According to the multi-enzyme relationship (Eq. (S25) of the SI Text), we thus expect the intracellular substrate concentration to be higher than what would be "predicted" from FruK concentrations alone. This is indeed what we find: the observed concentrations are 4.0-fold and 12.7-fold higher, respectively, than "predicted" from Eq. (2) (Table S1).

\section{Discussion}

Estimating reaction costs through the total mass of the molecules involved can only provide a rough approximation to the real cellular expenditure. It is likely that for some metabolite-enzyme pairs, specific biophysical or biochemical constraints lead to further deviations from our predictions. First, natural selection may favour lower concentrations for toxic metabolites than those predicted here. Second, the concentration of metabolites that enter the cell through passive diffusion cannot exceed extracellular concentrations. Third, as examined in the Fumarase A example above, the optimal concentrations of metabolites according to Eq. (2) may provide an insufficient thermodynamic driving force for some reversible reactions, and the generalization given in Eq. (S50) of the SI Text will be more appropriate. Finally, we emphasize that our predictions will only hold for cells whose transcriptional regulation of enzymes and transporters has been optimized by natural selection for metabolic efficiency in the specific condition examined.

Because the same reaction rates can be achieved with different combinations of enzyme and metabolite concentrations (Fig. 1), current metabolic models that account for reaction kinetics are either highly underdetermined or must be constrained by experimental data (33). The 
organizing principle exemplified by Eq. (2) - a minimal summed mass concentration of solutes has the potential to massively reduce the complexity of such models, as it provides an objective function capable of distinguishing between alternative kinetic solutions (11). For enzymatic subsystems composed of effectively irreversible reactions, Eq. (2) and its generalizations provide one-to-one correspondences between reaction rates and dominant enzyme as well as metabolite concentrations; the substrate concentration of a non-dominant enzyme can be treated as a constant set by the substrate's dominant reaction, resulting again in a one-to-one correspondence between reaction rate and enzyme concentration. Such improved kinetic modelling, together with a general appreciation of the cellular organizing principle of minimal summed mass concentrations, will allow the design of more efficient cellular systems and will thereby facilitate further progress in synthetic biology (34).

It is commonly assumed that in vivo enzyme and metabolite concentrations are not governed by a general optimization principle, but are determined by the biochemical properties of each metabolite and of the enzymes by which it is consumed $(12,35,36)$; reaction rates are often assumed to be governed by enzyme concentrations alone $(2,9,14,16)$. In contrast, theoretical considerations of optimal enzyme properties (37) and large-scale modelling studies $(11,33)$ as well as metabolite (38) and flux (26) measurements and perturbation experiments (21) indicate that reaction rates are jointly determined by enzyme and metabolite concentrations. Our results explain and quantify this relationship: metabolite and dominant enzyme pools are tightly balanced according to a simple organizing principle, likely reflecting natural selection for the parsimonious use of cellular resources. 


\section{Materials and Methods}

\section{E. coli data}

We obtained Escherichia coli strain BW25113 enzyme concentrations (23) and metabolite concentrations (24) from two recent publications. Metabolite concentrations in $\mu \mathrm{mol} / \mathrm{gCDW}(24)$ were converted to cytosolic molar concentrations based on the same conversion factor between cytosol volume and cell dry weight $(2.3 \mathrm{ml} / \mathrm{gCDW})$ used by the original authors (24). Enzyme concentrations in protein mass/cell (23) were converted to cytosolic molar concentrations based on: (i) protein molecular weight (23); (ii) cell dry weight (CDW) estimated based on the relationship PDW/CDW=-0.27869 $\mu+0.64034$, derived from Table S1 in Ref. (39), with growth rate $\mu$ (in units of $1 / \mathrm{h}$ ) and total protein dry weight (PDW) measured in each condition (23); (iii) the same conversion factor between cytosol volume and cell dry weight $(2.3 \mathrm{ml} / \mathrm{gCDW})$ used for the metabolite concentrations (24). Metabolite molecular weights were obtained from EcoCyc (40).

We collected the Michaelis constants $\left(K_{m}\right)$ of wild-type enzymes from EcoCyc (40), BRENDA (41), and UniProt (42). All experimental values are from E. coli, with the exception of four metabolite-enzyme pairs where only data from other organisms are available: D-ribulose 5phosphate-ribose-5-phosphate isomerase A (Ru5P-rpiA), 1,3-bisphospho-D-glyceratephosphoglycerate kinase (13DGP-pgk), ADP-phosphoglycerate kinase (ADP-pgk), and glycerone phosphate-fructose bisphosphate aldolase (DHAP-fbaA); we did not consider $K_{m}$ values of the extremophile Sulfolobus solfataricus, as these were obtained from measurements at $70^{\circ} \mathrm{C}$. If more than one $K_{m}$ was listed across the databases (Table S1), we first checked if these values were mostly within the same order of magnitude (i.e., if the geometric standard deviation was $\leq 10$ ); in this case, we used the geometric mean of all available values. Otherwise, we considered the available data for $K_{m}$ to be too unreliable to be included. The data for pairs of metabolites and dominant enzymes is listed in Table S1.

\section{S. cerevisiae data}

We obtained metabolite concentration data from Ref. (26), in which Saccharomyces cerevisiae derived from prototrophic strains $\mathrm{S} 288 \mathrm{C}$ and $\mathrm{W} 303$ were grown at $30{ }^{\circ} \mathrm{C}$ in $2 \% \mathrm{w} / \mathrm{v}$ glucose medium containing Yeast Nitrogen Base (YNB) without amino acids. Enzyme concentration data is from Ref. (25), in which S. cerevisiae strain BY4741 was grown at $30{ }^{\circ} \mathrm{C}$ in $2 \% \mathrm{w} / \mathrm{v}$ 
glucose supplemented with YPD medium (with amino acids). The Enzyme concentrations in molecules/cell were converted to cytosolic molar assuming cytosol volume of $21 \mathrm{fl}$, based on: (i) average cell volume of $42 \mathrm{fl}$ when growing on YPD medium Ref. (43); (ii) cytosol volume is about half of the cell volume Ref. (44). Metabolite molecular weights were obtained from BioCyc. Michaelis constants $K_{m}$ were collected from Ref. (26), as they are the geometric mean of all available values in BRENDA for $S$. cerevisiae; we confirmed that in each case, the geometric standard deviation was $<10$, i.e., all reported values were of the same order of magnitude. In almost all cases were no $K_{m}$ value from $S$. cerevisiae was available, there were also no measurements from other organisms. The $K_{m}$ for fumarate-URA1 (dihydroorotate dehydrogenase) is missing from BRENDA and from Ref. (26), and was obtained from BioCyc instead. The data for pairs of metabolites and dominant enzymes is listed in Table S1.

The genomes of the yeast strains S288C/W303 and BY4741 are highly similar (45), so that their intracellular concentrations of enzymes and metabolites are likely to be comparable if assayed under the same conditions. However, the two growth media employed by Park et al. (26) and Kulak et al. (25) differ substantially: while YNB (26) is a defined medium that contains no amino acids, YPD (25) contains peptone and yeast extract, making it rich in amino acids. The different growth conditions are likely to induce different intracellular concentrations of enzymes and metabolites, especially of those molecule types involved in amino acid synthesis. Accordingly, we expect to see more deviations between predicted and measured concentrations in yeast than in the other cell types examined; however, no better matching absolute enzyme and metabolite concentration data is available.

\section{Red blood cell data}

We obtained molecular weights, number of binding sites, and binding site concentrations of enzymes, as well as metabolite concentrations for red blood cells from Ref. (27). Albe et al. (27) considered fructose 6-phosphate to be the substrate of pgi; we changed this to glucose 6phosphate in agreement with the direction of glycolysis. Enzyme concentrations were determined dividing the binding site concentration by the number of binding sites of each enzyme. $K_{m}$ values were obtained from BRENDA. The values are listed in Table S1.

\section{Green alga data}

We obtained the molar concentration of Rubisco binding sites and its substrate ribulose-1,5biphosphate (RuBP) in the green alga Chlamydomonas reinhardtii from Ref. (28). We calculated 
the Rubisco molar concentration dividing the reported binding site concentration by the number of binding sites according to BRENDA. We considered the concentrations during steady-state in the wild type cell under constant low light intensity (28), using the geometric mean for RuBP concentration (which was measured twice). The molecular weight of $C$. reinhardtii Rubisco was obtained from BRENDA, and the $K_{m}$ for RuBP from Ref. (46). The values are listed in Table S1.

\section{Identification of dominant enzymes in E. coli}

For an automated identification of dominant enzymes, we used the sybil and sybilSBML (47) packages in R (48), with the EcoCyc metabolic model for E. coli exported as an SBML file using Pathway Tools 19.5 (49). For each metabolite measured in Ref. (24), we first identified all reactions using it as a substrate according to the metabolic model. The gene-reaction associations given in the EcoCyc model through b-numbers were used to map the reactions to the proteins measured in Ref. (23), identified by P-numbers. The concentration of enzymes was determined from the protein concentrations and the enzyme protein stoichiometries obtained from Ref. (50) (assuming stoichiometries of 1 for enzymes not listed in (50)).

For each substrate assayed in Ref. (24), we determined a dominance score (hereafter referred to simply as "dominance") for each enzyme consuming it that was assayed in Ref. (23). The dominance of an enzyme was defined as the fraction it contributes to the total mass concentration of all assayed enzymes using the substrate. An enzyme was considered "dominant" for the substrate if its dominance was $>0.5$, i.e., its molecules constituted more than half of the total protein mass consuming the substrate. We only attempted to assess dominance if more than half of the enzymes consuming a given substrate were assayed in Ref. (23). We excluded membranebound and periplasmic enzymes based on Gene Ontology annotations (51) (GO categories 0016020 (membrane), 0005886 (plasma membrane), 0005887 (integral component of plasma membrane), 0042597 (periplasmic space)), as in these cases the estimated enzyme concentrations will not correspond to actual cytosolic concentrations. If the reaction catalyzed by the dominant enzyme was reversible according to the EcoCyc model, this substrate-enzyme pair was only considered further if the flux through the reaction was measured in the corresponding direction in Ref. (24). Cyclic AMP (cAMP) was not included in the analysis, as the major role of cAMP is not metabolic. cAMP regulates transcription through varying concentrations of cAMP-CPR; 
accordingly, the only enzyme using it as a substrate (cAMP phosphodiesterase) is unlikely to have a major impact on cAMP concentrations.

\section{Identification of dominant enzymes in S. cerevisiae}

We determined dominant enzymes in $S$. cerevisiae using the same automatic procedure as for $E$. coli, using the Yeast v. 7.6 model Ref. (52) (yeast.sourceforge.net) in SBML format. For each metabolite measured in Ref. (26), we first identified all reactions using it as a substrate according to the metabolic model. The concentration of enzymes was determined from the protein concentrations in Ref. (25), assuming stoichiometries of 1 for enzyme complexes.

For each substrate assayed in Ref. (26), an enzyme was considered "dominant" if its dominance was $>0.5$. We only attempted to assess dominance if more than half of the enzymes consuming a given substrate were assayed in Ref. (25). We excluded membrane-bound and periplasmic enzymes based on Gene Ontology annotations (51) (GO categories 0016020 (membrane), 0005886 (plasma membrane), 0005887 (integral component of plasma membrane), 0042597 (periplasmic space)), as in these cases the estimated enzyme concentrations will not correspond to actual cytosolic concentrations. For the same reason, we only considered the enzymes dominant if they are located in the "cytoplasm" compartment in the metabolic model and assigned as a component of "cytoplasm" in the Yeast Genome Database Ref. (45). If the reaction catalyzed by the dominant enzyme was reversible according to the BioCyc (53) S. cerevisiae model (the Yeast v. 7.6 model doesn't contain information about reversibility), this substrateenzyme pair was only considered further if the flux through the reaction was measured in the corresponding direction in Ref. (26).

\section{Identification of dominant enzymes in red blood cells and green algae}

We used the HumanCyc (54) database to identify the enzymes involved in consuming each metabolite measured in Ref. (27). High-throughput enzyme MS/MS measurements and molecular weights for human red blood cells were obtained from Ref. (55) (Table S1). As in the E. coli and S. cerevisiae analysis, we attempted to determine dominance only for those substrates for which more than half of the consuming enzymes were assayed. Enzymes were considered dominant if their dominance score was $>0.5$. According to the BioCyc database, RubisCO is the only enzyme consuming D-ribulose-1,5-bisphosphate (RuBP) in Chlamydomonas reinhardtii, so it is the dominant enzyme by default. 


\section{Empirical P-value}

To test if the predictions for the in silico cell model are statistically significantly better when using the molecular mass ratio $a_{m}$ than when using molecular yield for the limiting nutrient, $\mathrm{N}$, we estimated an empirical $P$-value as follows. As a null model, we assumed that both sets of predictions come from the same distribution (i.e., both predictions are equally good). We randomly re-assigned the two predictions using $a_{m}$ and $a_{N}$ for each observed data point to two groups and calculated the difference in geometric mean fold-error ( GMFE) between the groups; this was repeated $n=10^{\prime} 000$ times. In all of the 10'000 repetitions, the GMFE difference was smaller than the GMFE difference observed in the in silico cell simulations (0.15). The randomized GMFE differences were normally distributed. Accordingly, we estimated the $P$ value based on the mean value $\left(2.32 \times 10^{-6}\right)$ and the standard deviation $\left(2.19 \times 10^{-4}\right)$ of the randomized GMFE differences. The z-score of the GMFE difference observed in the in silico cell simulations is 68 , and thus $\mathrm{P}<10^{-15}$.

\section{Generation of Figure 1}

The reaction rate $v$ of the GMP reductase reaction (GMP-guaC) in E. coli growing on glycerol (green dot in Fig. 1) was calculated through the corresponding Michaelis-Menten equation (Eq. (S1)), assuming saturation of the enzyme with the other substrates, NADPH and H+, the experimentally determined concentrations of enzyme $\left(4.69 \times 10^{-7}\right.$ Molar) (23) and metabolite $\left(6.58 \times 10^{-5}\right.$ Molar) (24), and the kinetic parameters $k_{c a t}=0.28 \mathrm{~s}^{-1}, K_{m}=2.30 \times 10^{-6}$ Molar obtained from the EcoCyc and BRENDA databases (the $K_{m}$ value is the geometric mean over the available values, SI Table 1). The optimal enzyme concentration (circle in Fig. 1) was calculated using Eq. (2) with the GMP/guaC molecular weight ratio $a_{\mathrm{m}}=413.76$. The curve corresponds to Eq. (S3), with the colour code representing the summed mass concentration of GMP and guaC (Eq. (S4) with molecular weights $\left.m_{E}=149454.2 \mathrm{Da}, m_{M}=361.21 \mathrm{Da}\right)$.

\section{Acknowledgments}

Deniz Sezer shared important insights into the interpretation of Eq. (2). We thank Peer Bork, Oliver Ebenhöh, David Heckmann, Markus Kollmann, Tabea Mettler-Altmann, Balazs Papp, Daniel Rickert, and Itai Yanai for helpful discussions. This work was supported by the German Research Foundation (DFG grants IRTG 1515 supporting HD; FOR 1186 to VGM; EXC 1028 to VGM and MJL; CRC 680 to MJL). 


\section{References}

1. Berkhout J, Bruggeman FJ, \& Teusink B (2012) Optimality principles in the regulation of metabolic networks. Metabolites 2(3):529-552.

2. Bordbar A, Monk JM, King ZA, \& Palsson BO (2014) Constraint-based models predict metabolic and associated cellular functions. Nat Rev Genet 15(2):107-120.

3. Scott M, Klumpp S, Mateescu EM, \& Hwa T (2014) Emergence of robust growth laws from optimal regulation of ribosome synthesis. Mol Syst Biol 10:747.

4. Zhuang K, Vemuri GN, \& Mahadevan R (2011) Economics of membrane occupancy and respiro-fermentation. Mol Syst Biol 7.

5. Schuster S \& Heinrich R (1991) Minimization of Intermediate Concentrations as a Suggested Optimality Principle for Biochemical Networks .1. Theoretical-Analysis. $J$ Math Biol 29(5):425-442.

6. Atkinson DE (1969) Limitation of metabolite concentrations and the conservation of solvent capacity in the living cell. Current Topics in Cellular Regulation 1.

7. Dekel E \& Alon U (2005) Optimality and evolutionary tuning of the expression level of a protein. Nature 436(7050):588-592.

8. Stoebel DM, Dean AM, \& Dykhuizen DE (2008) The cost of expression of Escherichia coli lac operon proteins is in the process, not in the products. Genetics 178(3):1653-1660.

9. Basan M, et al. (2015) Overflow metabolism in Escherichia coli results from efficient proteome allocation. Nature 528(7580):99-+.

10. Kafri M, Metzl-Raz E, Jona G, \& Barkai N (2016) The Cost of Protein Production. Cell Rep 14(1):22-31.

11. Noor E, et al. (2016) The Protein Cost of Metabolic Fluxes: Prediction from Enzymatic Rate Laws and Cost Minimization. PLoS Comput Biol 12(11):e1005167.

12. Tepper N, et al. (2013) Steady-state metabolite concentrations reflect a balance between maximizing enzyme efficiency and minimizing total metabolite load. PLoS One 8(9):e75370.

13. Neidhardt FC \& Curtiss R (1996) Escherichia coli and Salmonella : cellular and molecular biology (ASM Press, Washington, D.C.) 2nd Ed.

14. Beg QK, et al. (2007) Intracellular crowding defines the mode and sequence of substrate uptake by Escherichia coli and constrains its metabolic activity. Proc Natl Acad Sci US A 104(31):12663-12668.

15. Molenaar D, van Berlo R, de Ridder D, \& Teusink B (2009) Shifts in growth strategies reflect tradeoffs in cellular economics. Mol Syst Biol 5:323.

16. Shlomi T, Benyamini T, Gottlieb E, Sharan R, \& Ruppin E (2011) Genome-scale metabolic modeling elucidates the role of proliferative adaptation in causing the Warburg effect. PLoS Comput Biol 7(3):e1002018.

17. Liebermeister W \& Klipp E (2006) Bringing metabolic networks to life: convenience rate law and thermodynamic constraints. Theor Biol Med Model 3:41. 
18. Scott M, Gunderson CW, Mateescu EM, Zhang Z, \& Hwa T (2010) Interdependence of cell growth and gene expression: origins and consequences. Science 330(6007):10991102.

19. Wegkamp A, et al. (2010) Physiological responses to folate overproduction in Lactobacillus plantarum WCFS1. Microb Cell Fact 9:100.

20. Glick BR (1995) Metabolic load and heterologous gene expression. Biotechnol Adv 13(2):247-261.

21. Fendt SM, et al. (2010) Tradeoff between enzyme and metabolite efficiency maintains metabolic homeostasis upon perturbations in enzyme capacity. Mol Syst Biol 6:356.

22. Lee B (1983) Calculation of Volume Fluctuation for Globular Protein Models. P Natl Acad Sci USA 80(2):622-626.

23. Schmidt A, et al. (2016) The quantitative and condition-dependent Escherichia coli proteome. Nat Biotechnol 34(1):104-110.

24. Gerosa L, et al. (2015) Pseudo-transition Analysis Identifies the Key Regulators of Dynamic Metabolic Adaptations from Steady-State Data. Cell Syst 1(4):270-282.

25. Kulak NA, Pichler G, Paron I, Nagaraj N, \& Mann M (2014) Minimal, encapsulated proteomic-sample processing applied to copy-number estimation in eukaryotic cells. Nat Methods 11(3):319-324.

26. Park JO, et al. (2016) Metabolite concentrations, fluxes and free energies imply efficient enzyme usage. Nat Chem Biol 12(7):482-489.

27. Albe KR, Butler MH, \& Wright BE (1990) Cellular Concentrations of Enzymes and Their Substrates. $J$ Theor Biol 143(2):163-195.

28. Mettler T, et al. (2014) Systems Analysis of the Response of Photosynthesis, Metabolism, and Growth to an Increase in Irradiance in the Photosynthetic Model Organism Chlamydomonas reinhardtii. The Plant cell 26(6):2310-2350.

29. O'Brien EJ, Utrilla J, \& Palsson BO (2016) Quantification and Classification of E. coli Proteome Utilization and Unused Protein Costs across Environments. PLoS Comput Biol 12(6):e1004998.

30. Schuetz R, Zamboni N, Zampieri M, Heinemann M, \& Sauer U (2012) Multidimensional optimality of microbial metabolism. Science 336(6081):601-604.

31. Weed RI, Berg G, \& Reed CF (1963) Is Hemoglobin an Essential Structural Component of Human Erythrocyte Membranes. J Clin Invest 42(4):581-\&.

32. Flint DH (1994) Initial kinetic and mechanistic characterization of Escherichia coli fumarase A. Arch Biochem Biophys 311(2):509-516.

33. Chakrabarti A, Miskovic L, Soh KC, \& Hatzimanikatis V (2013) Towards kinetic modeling of genome-scale metabolic networks without sacrificing stoichiometric, thermodynamic and physiological constraints. Biotechnol J 8(9):1043-U1105.

34. Weber W \& Fussenegger M (2012) Emerging biomedical applications of synthetic biology. Nature Reviews Genetics 13(1):21-35.

35. Liebermeister W (2005) Predicting physiological concentrations of metabolites from their molecular structure. J Comput Biol 12(10):1307-1315. 
36. Bar-Even A, Noor E, Flamholz A, Buescher JM, \& Milo R (2011) Hydrophobicity and charge shape cellular metabolite concentrations. PLoS Comput Biol 7(10):e1002166.

37. Cornish-Bowden A (1976) The effect of natural selection on enzymic catalysis. $J$ Mol Biol 101(1):1-9.

38. Bennett BD, et al. (2009) Absolute metabolite concentrations and implied enzyme active site occupancy in Escherichia coli. Nat Chem Biol 5(8):593-599.

39. Valgepea K, Adamberg K, Seiman A, \& Vilu R (2013) Escherichia coli achieves faster growth by increasing catalytic and translation rates of proteins. Mol Biosyst 9(9):23442358.

40. Keseler IM, et al. (2013) EcoCyc: fusing model organism databases with systems biology. Nucleic Acids Res 41(D1):D605-D612.

41. Chang A, et al. (2015) BRENDA in 2015: exciting developments in its 25th year of existence. Nucleic Acids Res 43(D1):D439-D446.

42. UniProt C (2015) UniProt: a hub for protein information. Nucleic Acids Res 43(Database issue):D204-212.

43. Jorgensen P, Nishikawa JL, Breitkreutz BJ, \& Tyers M (2002) Systematic identification of pathways that couple cell growth and division in yeast. Science 297(5580):395-400.

44. Uchida M, et al. (2011) Quantitative analysis of yeast internal architecture using soft Xray tomography. Yeast 28(3):227-236.

45. Cherry JM, et al. (2012) Saccharomyces Genome Database: the genomics resource of budding yeast. Nucleic Acids Res 40(Database issue):D700-705.

46. Spreitzer RJ, Thow G, \& Zhu GH (1995) Pseudoreversion Substitution at Large-Subunit Residue-54 Influences the Co2/O-2 Specificity of Chloroplast Ribulose-Bisphosphate Carboxylase Oxygenase. Plant Physiol 109(2):681-685.

47. Gelius-Dietrich G, Desouki AA, Fritzemeier CJ, \& Lercher MJ (2013) Sybil--efficient constraint-based modelling in R. BMC Syst Biol 7:125.

48. $\quad \mathrm{R}$ Core Team (2014) $R$ : A Language and Environment for Statistical Computing (R Foundation for Statistical Computing, Vienna, Austria).

49. Karp PD, et al. (2016) Pathway Tools version 19.0 update: software for pathway/genome informatics and systems biology. Brief Bioinform 17(5):877-890.

50. Liu JK, et al. (2014) Reconstruction and modeling protein translocation and compartmentalization in Escherichia coli at the genome-scale. BMC Syst Biol 8:110.

51. Gene Ontology C (2015) Gene Ontology Consortium: going forward. Nucleic Acids Res 43(Database issue):D1049-1056.

52. Aung HW, Henry SA, \& Walker LP (2013) Revising the Representation of Fatty Acid, Glycerolipid, and Glycerophospholipid Metabolism in the Consensus Model of Yeast Metabolism. Ind Biotechnol (New Rochelle N Y) 9(4):215-228.

53. Caspi R, et al. (2016) The MetaCyc database of metabolic pathways and enzymes and the BioCyc collection of pathway/genome databases. Nucleic Acids Res 44(D1):D471-480.

54. Romero P, et al. (2005) Computational prediction of human metabolic pathways from the complete human genome. Genome Biol 6(1):R2. 
bioRxiv preprint doi: https://doi.org/10.1101/128009; this version posted April 17, 2017. The copyright holder for this preprint (which was not certified by peer review) is the author/funder. All rights reserved. No reuse allowed without permission.

55. Roux-Dalvai F, et al. (2008) Extensive Analysis of the Cytoplasmic Proteome of Human Erythrocytes Using the Peptide Ligand Library Technology and Advanced Mass Spectrometry. Molecular \& Cellular Proteomics 7(11):2254-2269. 